J. Nonlinear Var. Anal. 3 (2019), No. 1, pp. 87-106

Available online at http://jnva.biemdas.com

https://doi.org/10.23952/jnva.3.2019.1.09

\title{
TWO NEW ALGORITHMS FOR FINDING A COMMON ZERO OF ACCRETIVE OPERATORS IN BANACH SPACES
}

\author{
TRUONG MINH TUYEN ${ }^{1, *}$, NGUYEN MINH TRANG ${ }^{2}$ \\ ${ }^{1}$ Department of Mathematics and Informatics, Thainguyen University of Sciences, Thai Nguyen, Vietnam \\ ${ }^{2}$ Faculty of International training, Thainguyen University of Technology, Thai Nguyen, Vietnam
}

\begin{abstract}
The purpose of this paper is to introduce two new algorithms which are based on Mann type steepest-descent methods for solving variational inequality problems over the set of common zeros of a finite family of $m$-accretive operators in Banach spaces.
\end{abstract}

Keywords. Accretive operator; Iterative algorithm; Steepest descent method; Variational inequality; Zero point.

2010 Mathematics Subject Classification. 47H06, 47H09, 47H10, 47J25.

\section{INTRODUCTION}

Let $H$ be a real Hilbert space. We use symbols $\langle.,$.$\rangle and \|$.$\| to denote the inner product and the norm$ in $H$, respectively. Let $C$ be a nonempty closed convex subset of $H$ and let $F: C \longrightarrow H$ be a nonlinear mapping. A variational inequality problem, denoted by $\mathrm{VI}(F, C)$, is to find a point $x^{*} \in C$ such that

$$
\left\langle F\left(x^{*}\right), x-x^{*}\right\rangle \geq 0, \forall x \in C .
$$

There are many problems of mathematics can be recast in terms of the problem of finding a solution of the variational inequality, for instance, partial differential equations, optimal control, optimization, mathematical programming, mechanics and finance; see [9] and the references therein.

In 2001, Yamada [18] introduced the hybrid steepest-descent method for solving problem (1.1), where $F: H \longrightarrow H$ is Lipschitz and strongly monotone operator and $C$ is the set of fixed points of a nonexpansive mapping $T: H \longrightarrow H$, i.e., $C=\operatorname{Fix}(T)$. Moreover, in this paper, Yamada also considered problem (1.1) in the case that $C$ is the set of common fixed points of a finite family of nonexpansive mappings $T_{1}, T_{2}, \ldots, T_{N}$, i.e., $C=\cap_{i=1}^{N} \operatorname{Fix}\left(T_{i}\right)$. He proved the following theorem.

Theorem 1.1. [18, Theorem 3.3] Let $T_{i}: H \longrightarrow H, i=1,2, \ldots, N$ be nonexpansive mappings with $C=\cap_{i=1}^{N} \operatorname{Fix}\left(T_{i}\right) \neq \emptyset$ and

$$
C=\operatorname{Fix}\left(T_{N} \ldots T_{1}\right)=\operatorname{Fix}\left(T_{1} T_{N} \ldots T_{2}\right)=\ldots=\operatorname{Fix}\left(T_{N-1} \ldots T_{1} T_{N}\right) .
$$

Suppose that a mapping $F: H \longrightarrow H$ is $k$-Lipschitz and $\eta$-strongly monotone over $\triangle=\cup_{i=1}^{N} T_{i}(H)$. With any $u_{0} \in H$, any $\mu \in\left(0,2 \eta / k^{2}\right)$, and any sequence $\left\{\lambda_{n}\right\} \subset(0,1)$ satisfying

${ }^{*}$ Corresponding author.

E-mail addresses: tuyentm@tnus.edu.vn (T.M. Tuyen), nguyenminhtrang@tnut.edu.vn (N.M. Trang).

Received December 11, 2018; Accepted February 14, 2019.

(C)2019 Journal of Nonlinear and Variational Analysis 
(L1) $\lim _{n \rightarrow \infty} \lambda_{n}=0$;

(L2) $\sum_{n=1}^{\infty} \lambda_{n}=\infty$;

(L3) $\sum_{n=1}^{\infty}\left|\lambda_{n+N}-\lambda_{n}\right|<\infty$,

the sequence $\left\{u_{n}\right\}$ generated by

$$
u_{n+1}=\left(I-\lambda_{n+1} \mu F\right) T_{[n+1]} u_{n}
$$

converges strongly to a unique solution of problem $\mathrm{VI}(C, F)$.

Let $E$ be a real Banach space and let $C$ be a nonempty closed convex subset of $E$. Let $F: E \longrightarrow E$ be a mapping. We consider the following variational inequality in the setting of Banach spaces: Find an element $x^{*} \in E$ such that

$$
\left\langle F\left(x^{*}\right), j\left(x-x^{*}\right)\right\rangle \geq 0, \forall x \in C .
$$

This problem is denoted by $\mathrm{VI}^{*}(F, C)$ in this paper.

Problem $\mathrm{VI}^{*}(F, C)$ for an inverse-strongly accretive operator $F$ over a nonempty closed and convex subset $C$ of a uniformly convex and 2-uniformly smooth Banach space $E$ has already been presented by Aoyama, Iiduka and Takahahsi in [1,2]. This problem with $F=I-f$, where $f$ is a contractive mapping and $C$ is set of zeros or common zeros of accretive operators in Banach spaces, is also studied by many authors, see, for example, $[6,7,8,11,14,15,19]$ and the references therein.

In 2008, Ceng, Ansari and Yao [5] studied problem $\mathrm{VI}^{*}(F, C)$ with $C$ is the set of fixed points of a nonexpansive mapping or $C$ is the set of zeros of an accretive operator in Banach spaces. They proved the following propositions.

Proposition 1.1. Let $E$ be a real reflexive and strictly convex Banach space with a uniformly Gâteaux differentiable norm. Suppose that $T: E \longrightarrow E$ is a continuous pseudocontractive mapping and $S=$ $\operatorname{Fix}(T) \neq \emptyset$. Assume that $F: E \longrightarrow E$ is $\delta$-strongly accretive and $\lambda$-strictly pseudocontractive with $\delta+\lambda>1$. For each $t \in(0,1)$, choose a number $\mu_{t} \in(0,1)$ arbitrarily and let $\left\{x_{t}\right\}$ be defined by

$$
x_{t}=t\left(I-\mu_{t} F\right)\left(x_{t}\right)+(1-t) T\left(x_{t}\right) .
$$

Then as $t \longrightarrow 0^{+},\left\{x_{t}\right\}$ converges strongly to a unique solution $u^{*}$ of $\operatorname{VI}^{*}(F, C)$.

Proposition 1.2. Let $E$ be a real Banach space with a uniformly Gâteaux differentiable norm. Suppose that $T: E \longrightarrow E$ is a continuous pseudocontractive mapping and $S=\operatorname{Fix}(T) \neq \emptyset$. Assume that $F: E \longrightarrow$ $E$ is $\delta$-strongly accretive and $\lambda$-strictly pseudocontractive with $\delta+\lambda>1$. If there exists a bounded sequence $\left\{x_{n}\right\}$ such that $\lim _{n \rightarrow \infty}\left\|x_{n}-T\left(x_{n}\right)\right\|=0$ and $u^{*}=\lim _{t \rightarrow 0^{+}} x_{t}$, where $\left\{x_{t}\right\}$ is defined by (1.4), then

$$
\limsup _{n \rightarrow \infty}\left\langle F\left(u^{*}\right), j\left(u^{*}-x_{n}\right)\right\rangle \leq 0 .
$$

When $C=A^{-1} 0$, with an $m$-accretive operator $A: E \longrightarrow 2^{E}$ in an uniformly smooth Banach space $E$, and the steepest-descent method, Ceng, Ansari and Yao [5] introduced the following iterative method

$$
\left\{\begin{array}{l}
y_{n}=\alpha_{n} x_{n}+\left(1-\alpha_{n}\right) J_{r_{n}}^{A} x_{n} \\
x_{n+1}=\left(I-\lambda_{n} F\right)\left(y_{n}\right)
\end{array}\right.
$$

to solve problem $\operatorname{VI}^{*}(F, C)$, where $F: E \longrightarrow E$ is $\delta$-strongly accretive and $\lambda$-strictly pseudocontractive with $\delta+\lambda>1$. They proved that, if the sequences of positive real numbers $\left\{\alpha_{n}\right\},\left\{r_{n}\right\}$ and $\left\{\lambda_{n}\right\} \subset(0,1)$ satisfy the following conditions 
C1) $\lim _{n \rightarrow \infty} \lambda_{n}=0, \sum_{n=0}^{\infty} \lambda_{n}=\infty$,

C2) $r_{n} \geq \varepsilon>0$ for all $n,\left\{\alpha_{n}\right\} \subset(a, b)$ for some $a, b \in(0,1)$,

C3) $\sum_{n=0}^{\infty}\left|\alpha_{n+1}-\alpha_{n}\right|<\infty, \sum_{n=0}^{\infty}\left|\lambda_{n+1}-\lambda_{n}\right|<\infty$ and $\sum_{n=0}^{\infty}\left|r_{n+1}-r_{n}\right|<\infty$,

then the sequence $\left\{x_{n}\right\}$ generated by (1.5) converges strongly to an element $x^{*} \in C$ which is the unique solution of $\mathrm{VI}^{*}(F, C)$.

In this paper, we introduce two new algorithms which are the extensions of iterative method (1.5) for problem $\mathrm{VI}^{*}(F, C)$, where $C$ is the set of common zeros of a finite family of $m$-accretive operators in a uniformly convex Banach space. Moreover, we also show that conditions C1) and C2) above are sufficient to ensure the strong convergence of the iterative method. In Section 4, we give an application of the main result for the problem of finding a common fixed point of nonexpansive mappings. Finally, in Section 5, a numerical example is given to illustrate the main result and to show its performance.

\section{PRELIMINARIES}

Let $E$ be a real Banach space with norm $\|$.$\| and let E^{*}$ be its dual. The value of $f \in E^{*}$ at $x \in E$ is denoted by $\langle x, f\rangle$. Let $\left\{x_{n}\right\}$ be a sequence in $E$. $x_{n} \longrightarrow x$ (resp. $x_{n} \rightarrow x, x_{n} \stackrel{*}{\rightarrow} x$ ) denotes the strong (resp. weak, weak*) convergence of the sequence $\left\{x_{n}\right\}$ to $x$.

Let $J$ denote the normalized duality mapping from $E$ into $2^{E^{*}}$ given by

$$
J(x)=\left\{f \in E^{*}:\langle x, f\rangle=\|x\|^{2}=\|f\|^{2}\right\}, \forall x \in E,
$$

where $\langle.,$.$\rangle denotes the generalized duality pairing. It is well known that if E^{*}$ is strictly convex, then $J$ is single-valued. In the sequel, we denote the single-valued normalized duality mapping by $j$.

We always use $S_{E}$ to denote the unit sphere $S_{E}=\{x \in E:\|x\|=1\}$ and $\operatorname{Fix}(T)$ to denote the set of the fixed point of the mapping $T: C \subseteq E \longrightarrow E$, i.e., $\operatorname{Fix}(T)=\{x \in C: T(x)=x\}$.

A Banach space $E$ is said to be strictly convex if

$$
x, y \in S_{E} \text { with } x \neq y \text {, implies that }\|(1-t) x+t y\|<1 \text { for all } t \in(0,1) .
$$

A Banach space $E$ is said to be uniformly convex if for any $\varepsilon \in(0,2]$ the inequalities $\|x\| \leq 1,\|y\| \leq$ $1,\|x-y\| \geq \varepsilon$ imply there exists a $\delta=\delta(\varepsilon)>0$ such that

$$
\frac{\|x+y\|}{2} \leq 1-\delta
$$

A Banach $E$ is said to be smooth provided the limit

$$
\lim _{t \rightarrow 0} \frac{\|x+t y\|-\|x\|}{t}
$$

exists for each $x$ and $y$ in $S_{E}$. In this case, the norm of $E$ is said to be Gâteaux differentiable. It is said to be uniformly Gâteaux differentiable if for each $y \in S_{E}$, this limit is attained uniformly for $x \in S_{E}$. It is well known that every uniformly smooth space has uniformly Gâteaux differentiable norm.

For an operator $A: E \longrightarrow 2^{E}$, we define its domain, range and graph as follows:

$$
\begin{aligned}
& D(A)=\{x \in E: A x \neq \emptyset\}, \\
& R(A)=\cup\{A z: z \in D(A)\},
\end{aligned}
$$

and

$$
G(A)=\{(x, y) \in E \times E: x \in D(A), y \in A x\}
$$


respectively. The inverse $A^{-1}$ of $A$ is defined by

$$
x \in A^{-1} y \text {, if and only if } y \in A x .
$$

The operator $A$ is said to be accretive if, for each $x, y \in D(A)$, there exists $j(x-y) \in J(x-y)$ such that $\langle u-v, j(x-y)\rangle \geq 0$ for all $u \in A x$ and $v \in A y$. We denote by $I$ the identity operator on $E$. An accretive operator $A$ is said to be maximal accretive if there is no proper accretive extension of $A$ and $m$-accretive if $R(I+\lambda A)=E$ for all $\lambda>0$. If $A$ is $m$-accretive, then it is maximal accretive, but the converse is not true in general. If $A$ is accretive, then we can define, for each $\lambda>0$, a nonexpansive single-valued mapping $J_{\lambda}^{A}: R(I+\lambda A) \longrightarrow D(A)$ by

$$
J_{\lambda}^{A}=(I+\lambda A)^{-1}
$$

which is called the resolvent of $A$. An accretive operator $A$ defined on a Banach space $E$ is said to satisfy the range condition if $\overline{D(A)} \subset R(I+\lambda A)$ for all $\lambda>0$, where $\overline{D(A)}$ denotes the closure of the domain of $A$. We know that for an accretive operator $A$ which satisfies the range condition, $A^{-1} 0=\operatorname{Fix}\left(J_{\lambda}^{A}\right)$ for all $\lambda>0$. It is easy to see that if $A$ is an $m$-accretive operator, then $A$ satisfies the range condition (see $[12,13])$.

Recall that a mapping $F: E \longrightarrow E$ is said to be $\delta$-strongly accretive if for each $x, y \in E$ there exists $j(x-y) \in J(x-y)$ such that

$$
\langle F(x)-F(y), j(x-y)\rangle \geq \delta\|x-y\|^{2}
$$

for some $\delta \in(0,1)$. A mapping $F: E \longrightarrow E$ is said to be $\lambda$-strictly pseudocontractive [4] if for each $x, y \in E$ there exists $j(x-y) \in J(x-y)$ such that

$$
\langle F(x)-F(y), j(x-y)\rangle \leq\|x-y\|^{2}-\lambda\|x-y-(F(x)-F(y))\|^{2}
$$

for some $\lambda \in(0,1)$. Recall that $F$ is said to be pseudocontractive if, for each $x, y \in E$, there exists $j(x-y) \in J(x-y)$ such that

$$
\langle F(x)-F(y), j(x-y)\rangle \leq\|x-y\|^{2} .
$$

So, if $F$ is a nonexpansive mapping, that is, $\|F(x)-F(y)\| \leq\|x-y\|$ for all $x, y \in E$, then $F$ is a pseudocontractive mapping.

Lemma 2.1. [16] $E$ is uniformly convex if and only if, for each $r>0$, there exists a continuous strictly increasing and convex function $\varphi: \mathbb{R}^{+} \longrightarrow \mathbb{R}^{+}$with $\varphi(0)=0$ such that

$$
\|\alpha x+(1-\alpha) y\|^{2} \leq \alpha\|x\|^{2}+(1-\alpha)\|y\|^{2}-\alpha(1-\alpha) \varphi(\|x-y\|),
$$

for all $x, y \in E$ with $\max \{\|x\|,\|y\|\} \leq r$ and $\alpha \in[0,1]$.

Lemma 2.2. [5] Let $E$ be a real smooth Banach space and let $F: E \longrightarrow E$ be a mapping. If $F$ is $\delta$ strongly accretive and $\lambda$ - strictly pseudocontractive with $\delta+\lambda>1$, then, for any fixed number $\tau \in(0,1]$, $I-\tau F$ is contractive with constant $1-\tau\left(1-\sqrt{\frac{1-\delta}{\lambda}}\right)$.

Lemma 2.3. [3] Let $A: D(A) \subset E \longrightarrow 2^{E}$ be an accretive operator. For $\lambda, \mu>0$, and $x \in E$, we have

$$
J_{\lambda}^{A} x=J_{\mu}^{A}\left(\frac{\mu}{\lambda} x+\left(1-\frac{\mu}{\lambda}\right) J_{\lambda}^{A} x\right) .
$$


Lemma 2.4. Let $A: D(A) \subset E \longrightarrow 2^{E}$ be an accretive operator. For $r \geq s>0$, we have

$$
\left\|x-J_{s}^{A} x\right\| \leq 2\left\|x-J_{r}^{A} x\right\|
$$

for all $x \in R(I+r A) \cap R(I+s A)$.

Proof. From Lemma 2.3, we have

$$
\begin{aligned}
\left\|x-J_{s}^{A} x\right\| & \leq\left\|x-J_{r}^{A} x\right\|+\left\|J_{r}^{A} x-J_{s}^{A} x\right\| \\
& =\left\|x-J_{r}^{A} x\right\|+\left\|J_{s}^{A}\left(\frac{s}{r} x+\left(1-\frac{s}{r}\right) J_{r}^{A} x\right)-J_{s}^{A} x\right\| \\
& \leq\left\|x-J_{r}^{A} x\right\|+\left(1-\frac{s}{r}\right)\left\|x-J_{r}^{A} x\right\| \\
& \leq 2\left\|x-J_{r}^{A} x\right\| .
\end{aligned}
$$

This completes the proof.

Lemma 2.5. [10] Let $\left\{s_{n}\right\}$ be a real sequence that does not decrease at infinity, in the sense that there exists a subsequence $\left\{s_{n_{k}}\right\}$ such that

$$
s_{n_{k}} \leq s_{n_{k}+1}, \forall k \geq 0 .
$$

For every $n>n_{0}$, define an integer sequence $\{\tau(n)\}$ as

$$
\tau(n)=\max \left\{n_{0} \leq k \leq n: s_{k}<s_{k+1}\right\} .
$$

Then $\tau(n) \rightarrow \infty$ as $n \rightarrow \infty$ for all $n>n_{0}$,

$$
\max \left\{s_{\tau(n)}, s_{n}\right\} \leq s_{\tau(n)+1} .
$$

Lemma 2.6. [17] Let $\left\{s_{n}\right\}$ be a sequence of nonnegative numbers. Let $\left\{\alpha_{n}\right\}$ be a sequence in $(0,1)$, and let $\left\{c_{n}\right\}$ be a sequence of real numbers satisfying the conditions

i) $s_{n+1} \leq\left(1-\alpha_{n}\right) s_{n}+\alpha_{n} c_{n}$,

ii) $\sum_{n=0}^{\infty} \alpha_{n}=\infty, \limsup _{n \rightarrow \infty} c_{n} \leq 0$.

Then $\lim _{n \rightarrow \infty} s_{n}=0$.

\section{MAin RESUlts}

Let $E$ be a real uniformly convex Banach space with a uniformly Gâteaux differential norm. Assume that $F: E \longrightarrow E$ is $\delta$-strongly accretive and $\lambda$-strictly pseudocontractive with $\delta+\lambda>1$. Let $A_{i}: E \longrightarrow$ $2^{E}, i=1,2, \ldots, N$, be $m$-accretive operators such that $S=\cap_{i=1}^{N} A_{i}^{-1} 0 \neq \emptyset$. We consider the following problem:

Find an element $p \in S$ which is a solution of $\operatorname{VI}^{*}(F, S)$.

3.1. A cyclic algorithm. First, in order to solve Problem (3.1), we propose the following cyclic algorithm.

Algorithm 3.1. For any $x_{0} \in E$, let $\left\{x_{n}\right\}$ be a sequence generated by

$$
\begin{aligned}
& y_{n}^{0}=x_{n}, n \geq 0, \\
& y_{n}^{i}=\left(1-\beta_{n}^{i}\right) y_{n}^{i-1}+\beta_{n}^{i} J_{i, n} y_{n}^{i-1}, i=1,2, \ldots, N, n \geq 0, J_{i, n}=J_{r_{n}^{i}}^{A_{i}}, \\
& x_{n+1}=\left(I-\lambda_{n} F\right)\left(y_{n}^{N}\right), n \geq 0,
\end{aligned}
$$


where $\left\{\lambda_{n}\right\},\left\{r_{n}^{i}\right\}$ and $\left\{\beta_{n}^{i}\right\}, i=1,2, \ldots, N$, are sequences of positive real numbers.

Now, we have the following theorem.

Theorem 3.1. Let $\left\{x_{n}\right\}$ be a sequence generated by Algorithm 3.1. If sequences $\left\{\lambda_{n}\right\},\left\{r_{n}^{i}\right\}$ and $\left\{\beta_{n}^{i}\right\}$, $i=1,2, \ldots, N$ satisfy the following conditions:

i) $\min _{i=1,2, \ldots, N}\left\{\inf _{n}\left\{r_{n}^{i}\right\}\right\} \geq r>0$ for all $i=1,2, \ldots, N$;

ii) $\left\{\beta_{n}^{i}\right\} \subset(\alpha, \beta)$ with $\alpha, \beta \in(0,1)$ for all $i=1,2, \ldots, N$;

iii) $\left\{\lambda_{n}\right\} \subset(0,1), \lim _{n \rightarrow \infty} \lambda_{n}=0, \sum_{n=0}^{\infty} \lambda_{n}=\infty$,

then $\left\{x_{n}\right\}$ converges strongly to an element $p \in S$, which is the unique solution of $\operatorname{VI}^{*}(F, S)$.

Proof. First, we show that the sequence $\left\{x_{n}\right\}$ is bounded.

Taking $u \in S$, we have

$$
\begin{aligned}
\left\|y_{n}^{N}-u\right\|= & \left\|\left(1-\beta_{n}^{i}\right) y_{n}^{i-1}+\beta_{n}^{i} J_{i, n} y_{n}^{i-1}-u\right\| \\
\leq & \left(1-\beta_{n}^{i}\right)\left\|y_{n}^{i-1}-u\right\|+\beta_{n}^{i}\left\|J_{i, n} y_{n}^{i-1}-u\right\| \\
\leq & \left(1-\beta_{n}^{i}\right)\left\|y_{n}^{i-1}-u\right\|+\beta_{n}^{i}\left\|y_{n}^{i-1}-u\right\| \\
= & \left\|y_{n}^{i-1}-u\right\| \\
& \vdots \\
\leq & \left\|y_{n}^{0}-u\right\|=\left\|x_{n}-u\right\| .
\end{aligned}
$$

Thus, by Lemma 2.2, we have

$$
\begin{aligned}
\left\|x_{n+1}-u\right\| & =\left\|\left(I-\lambda_{n} F\right)\left(y_{n}^{N}\right)-u\right\| \\
& =\left\|\left(I-\lambda_{n} F\right)\left(y_{n}^{N}\right)-\left(I-\lambda_{n} F\right)(u)-\lambda_{n} F(u)\right\| \\
& \leq\left\|\left(I-\lambda_{n} F\right)\left(y_{n}^{N}\right)-\left(I-\lambda_{n} F\right)(u)\right\|+\lambda_{n}\|F(u)\| \\
& \leq\left(1-\lambda_{n}\left(1-\sqrt{\frac{1-\delta}{\lambda}}\right)\right)\left\|y_{n}^{N}-u\right\|+\lambda_{n}\|F(u)\| \\
& \leq \max \left\{\left\|y_{n}^{N}-u\right\|,\left(1-\sqrt{\frac{1-\delta}{\lambda}}\right)^{-1}\|F(u)\|\right\} \\
& \leq \max \left\{\left\|x_{n}-u\right\|,\left(1-\sqrt{\frac{1-\delta}{\lambda}}\right)^{-1}\|F(u)\|\right\} .
\end{aligned}
$$

By induction, we get

$$
\left\|x_{n}-u\right\| \leq \max \left\{\left\|x_{0}-u\right\|,\left(1-\sqrt{\frac{1-\delta}{\lambda}}\right)^{-1}\|F(u)\|\right\}, \forall n \geq 0
$$

Thus, $\left\{x_{n}\right\}$ is bounded. $\left\{y_{n}^{i}\right\},\left\{F\left(y_{n}^{i}\right)\right\}, i=1,2, \ldots, N$ are also bounded. Let $p$ is the unique solution of $\mathrm{VI}^{*}(F, S)$, that is,

$$
\langle F(p), j(p-u)\rangle \leq 0, \forall u \in S
$$


From (3.2), we have

$$
\begin{aligned}
\left\|x_{n+1}-p\right\|^{2} & =\left\langle\left(I-\lambda_{n} F\right)\left(y_{n}^{N}\right)-p, j\left(x_{n+1}-p\right)\right\rangle \\
& =\left\langle\left(I-\lambda_{n} F\right)\left(y_{n}^{N}\right)-\left(I-\lambda_{n} F\right)(p), j\left(x_{n+1}-p\right)\right\rangle+\lambda_{n}\left\langle F(p), j\left(p-x_{n+1}\right)\right\rangle \\
& \leq\left(1-\lambda_{n}\left(1-\sqrt{\frac{1-\delta}{\lambda}}\right)\right)\left\|y_{n}^{N}-p\right\|\left\|x_{n+1}-p\right\|+\lambda_{n}\left\langle F(p), j\left(p-x_{n+1}\right)\right\rangle \\
& \leq\left(1-\lambda_{n}\left(1-\sqrt{\frac{1-\delta}{\lambda}}\right)\right) \frac{\left\|y_{n}^{N}-p\right\|^{2}+\left\|x_{n+1}-p\right\|^{2}}{2}+\lambda_{n}\left\langle F(p), j\left(p-x_{n+1}\right)\right\rangle .
\end{aligned}
$$

This implies that

$$
\begin{aligned}
\left\|x_{n+1}-p\right\|^{2} \leq & \frac{\left(1-\lambda_{n}\left(1-\sqrt{\frac{1-\delta}{\lambda}}\right)\right)}{1+\lambda_{n}\left(1-\sqrt{\frac{1-\delta}{\lambda}}\right)}\left\|y_{n}^{N}-p\right\|^{2} \\
& +\frac{2 \lambda_{n}}{1+\lambda_{n}\left(1-\sqrt{\frac{1-\delta}{\lambda}}\right)}\left\langle F(p), j\left(p-x_{n+1}\right)\right\rangle \\
= & \left(1-\frac{2 \lambda_{n}\left(1-\sqrt{\frac{1-\delta}{\lambda}}\right)}{1+\lambda_{n}\left(1-\sqrt{\frac{1-\delta}{\lambda}}\right)}\right)\left\|y_{n}^{N}-p\right\|^{2} \\
& +\frac{2 \lambda_{n}\left(1-\sqrt{\frac{1-\delta}{\lambda}}\right)}{1+\lambda_{n}\left(1-\sqrt{\frac{1-\delta}{\lambda}}\right)}\left(1-\sqrt{\frac{1-\delta}{\lambda}}\right)^{-1}\left\langle F(p), j\left(p-x_{n+1}\right)\right\rangle .
\end{aligned}
$$

From Lemma 2.1, we have

$$
\begin{aligned}
\left\|y_{n}^{N}-p\right\|^{2}= & \left\|\left(1-\beta_{n}^{N}\right) y_{n}^{N-1}+\beta_{n}^{N} J_{N, n} y_{n}^{N-1}-p\right\|^{2} \\
\leq & \left(1-\beta_{n}^{N}\right)\left\|y_{n}^{N-1}-p\right\|^{2}+\beta_{n}^{N}\left\|J_{N, n} y_{n}^{N-1}-p\right\|^{2} \\
& -\beta_{n}^{N}\left(1-\beta_{n}^{N}\right) \varphi\left(\left\|y_{n}^{N-1}-J_{N, n} y_{n}^{N-1}\right\|\right) \\
\leq & \left(1-\beta_{n}^{N}\right)\left\|y_{n}^{N-1}-p\right\|^{2}+\beta_{n}^{N}\left(\left\|y_{n}^{N-1}-p\right\|^{2}\right. \\
& -\alpha(1-\beta) \varphi\left(\left\|y_{n}^{N-1}-J_{N, n} y_{n}^{N-1}\right\|\right) \\
= & \left\|y_{n}^{N-1}-p\right\|^{2}-\alpha(1-\beta) \varphi\left(\left\|y_{n}^{N-1}-J_{N, n} y_{n}^{N-1}\right\|\right) \\
& \vdots \\
= & \left\|y_{n}^{0}-p\right\|^{2}-\alpha(1-\beta) \sum_{i=1}^{N} \varphi\left(\left\|y_{n}^{i-1}-J_{i, n} y_{n}^{i-1}\right\|\right) \\
= & \left\|x_{n}-p\right\|^{2}-\alpha(1-\beta) \sum_{i=1}^{N} \varphi\left(\left\|y_{n}^{i-1}-J_{i, n} y_{n}^{i-1}\right\|\right) .
\end{aligned}
$$


From (3.5) and (3.6), we obtain that

$$
\begin{aligned}
\left\|x_{n+1}-p\right\|^{2} \leq & \left(1-\frac{2 \lambda_{n}\left(1-\sqrt{\frac{1-\delta}{\lambda}}\right)}{1+\lambda_{n}\left(1-\sqrt{\frac{1-\delta}{\lambda}}\right)}\right)\left\|x_{n}-p\right\|^{2} \\
& +\frac{2 \lambda_{n}\left(1-\sqrt{\frac{1-\delta}{\lambda}}\right)}{1+\lambda_{n}\left(1-\sqrt{\frac{1-\delta}{\lambda}}\right)}\left(1-\sqrt{\frac{1-\delta}{\lambda}}\right)^{-1}\left\langle F(p), j\left(p-x_{n+1}\right)\right\rangle \\
& -\alpha(1-\beta)\left(1-\frac{2 \lambda_{n}\left(1-\sqrt{\frac{1-\delta}{\lambda}}\right)}{1+\lambda_{n}\left(1-\sqrt{\frac{1-\delta}{\lambda}}\right)}\right) \sum_{i=1}^{N} \varphi\left(\left\|y_{n}^{i-1}-J_{i, n} y_{n}^{i-1}\right\|\right) .
\end{aligned}
$$

Putting

and

$$
\begin{gathered}
s_{n}=\left\|x_{n}-p\right\|^{2}, \\
b_{n}=\frac{2 \lambda_{n}\left(1-\sqrt{\frac{1-\delta}{\lambda}}\right)}{1+\lambda_{n}\left(1-\sqrt{\frac{1-\delta}{\lambda}}\right)}
\end{gathered}
$$

$$
\begin{gathered}
c_{n}=\left(1-\sqrt{\frac{1-\delta}{\lambda}}\right)^{-1}\left\langle F(p), j\left(p-x_{n+1}\right)\right\rangle, \\
\sigma_{n}=\left(1-b_{n}\right) \alpha(1-\beta) \sum_{i=1}^{N} \varphi\left(\left\|y_{n}^{i-1}-J_{i, n} y_{n}^{i-1}\right\|\right),
\end{gathered}
$$

inequality (3.7) can be rewritten as

$$
s_{n+1} \leq\left(1-b_{n}\right) s_{n}+b_{n} c_{n}-\sigma_{n} .
$$

We will show that $s_{n} \rightarrow 0$ by considering two possible cases.

Case 1. $\left\{s_{n}\right\}$ is eventually decreasing, i.e., there exists $N_{0} \geq 0$ such that $\left\{s_{n}\right\}$ is decreasing for $n \geq N_{0}$ and thus $\left\{s_{n}\right\}$ must be convergent. It then follows from (3.8) that

$$
0 \leq \sigma_{n} \leq\left(s_{n}-s_{n+1}\right)+b_{n}\left(c_{n}-s_{n}\right) \rightarrow 0,
$$

which implies that

$$
\left\|y_{n}^{i-1}-J_{i, n} y_{n}^{i-1}\right\| \rightarrow 0
$$

for all $i=1,2, \ldots, N$.

Next, we will show that $\left\|x_{n}-J_{r}^{A_{i}} x_{n}\right\| \rightarrow 0$, for all $i=1,2, \ldots, N$.

Indeed, in the case that $i=1,\left\|x_{n}-J_{1, n} x_{n}\right\|=\left\|y_{n}^{0}-J_{1, n} y_{n}^{0}\right\| \rightarrow 0$. In the case that $i=2$, we have

$$
\begin{aligned}
\left\|x_{n}-J_{2, n} x_{n}\right\| & \leq\left\|x_{n}-y_{n}^{1}\right\|+\left\|y_{n}^{1}-J_{2, n} y_{n}^{1}\right\|+\left\|J_{2, n} y_{n}^{1}-J_{2, n} x_{n}\right\| \\
& \leq 2\left\|x_{n}-y_{n}^{1}\right\|+\left\|y_{n}^{1}-J_{2, n} y_{n}^{1}\right\| \\
& =2\left\|y_{n}^{0}-J_{1, n} y_{n}^{0}\right\|+\left\|y_{n}^{1}-J_{2, n} y_{n}^{1}\right\| \rightarrow 0 .
\end{aligned}
$$


So, we get $\left\|x_{n}-J_{2, n} x_{n}\right\| \rightarrow 0$. Similarly, we obtain $\left\|x_{n}-J_{i, n} x_{n}\right\| \rightarrow 0$ for all $i=3,4, \ldots, N$. By Lemma 2.2, $\left\|x_{n}-J_{r}^{A_{i}} x_{n}\right\| \leq 2\left\|x_{n}-J_{i, n} x_{n}\right\|$. So, $\left\|x_{n}-J_{r}^{A_{i}} x_{n}\right\| \rightarrow 0$ for all $i=1,2, \ldots, N$. Let $T=\frac{1}{N} \sum_{i=1}^{N} J_{r}^{A_{i}}$. Then $T$ is a nonexpansive mapping and $S=\operatorname{Fix}(T)$. From $\left\|x_{n}-T_{i}\left(x_{n}\right)\right\| \rightarrow 0$ for all $i=1,2, \ldots, N$ and by the following estimate

$$
\left\|x_{n}-T\left(x_{n}\right)\right\| \leq \frac{1}{N} \sum_{i=1}^{N}\left\|x_{n}-T_{i}\left(x_{n}\right)\right\|,
$$

we get $\lim _{n \rightarrow \infty}\left\|x_{n}-T\left(x_{n}\right)\right\|=0$. From Proposition 1.1 and Proposition 1.2, we obtain

$$
\limsup _{n \rightarrow \infty}\left\langle F(p), j\left(p-x_{n}\right)\right\rangle \leq 0 .
$$

Letting $K=\sup _{n}\left\{\left\|F\left(y_{n}^{N}\right)\right\|\right\}$, we have

$$
\begin{aligned}
\left\|x_{n+1}-x_{n}\right\| & =\left\|\left(I-\lambda_{n} F\right)\left(y_{n}^{N}\right)-x_{n}\right\| \\
\leq & \left\|\left(I-\lambda_{n} F\right)\left(x_{n}\right)-\left(I-\lambda_{n} F\right)\left(y_{n}^{N}\right)\right\|+\lambda_{n} K \\
\leq & \left(1-\lambda_{n}\left(1-\sqrt{\frac{1-\delta}{\lambda}}\right)\right)\left\|x_{n}-y_{n}^{N}\right\|+\lambda_{n} K \\
\leq & \left\|x_{n}-\left(1-\beta_{n}^{N}\right) y_{n}^{N-1}-\beta_{n}^{N} J_{N, n} y_{n}^{N-1}\right\|+\lambda_{n} K \\
\leq & \left\|x_{n}-y_{n}^{N-1}\right\|+\left\|y_{n}^{N-1}-J_{N, n} y_{n}^{N-1}\right\|+\lambda_{n} K \\
& \vdots \\
& \leq \sum_{i=1}^{N}\left\|y_{n}^{i-1}-J_{i, n} y_{n}^{i-1}\right\|+\lambda_{n} K,
\end{aligned}
$$

which implies that $\left\|x_{n+1}-x_{n}\right\| \rightarrow 0$, as $n \rightarrow \infty$. Thus, by (3.9) and the fact that the duality map $j$ is uniformly norm-to-weak* continuous on bounded set, we get

$$
\limsup _{n \rightarrow \infty}\left\langle F(p), j\left(p-x_{n+1}\right)\right\rangle \leq 0,
$$

that is, $\limsup _{n \rightarrow \infty} c_{n} \leq 0$. From (3.8), we have

$$
s_{n+1} \leq\left(1-b_{n}\right) s_{n}+b_{n} c_{n}
$$

and applying Lemma 2.6, we obtain $\lim _{n \rightarrow \infty} s_{n}=0$.

Case 2. $\left\{s_{n}\right\}$ is not eventually decreasing. Hence, there exists a subsequence $\left\{s_{n_{k}}\right\}$ of $\left\{s_{n}\right\}$ such that $s_{n_{k}} \leq s_{n_{k}+1}$ for all $k \geq 0$. By Lemma 2.3, we can define a subsequence $\left\{s_{\tau(n)}\right\}$ such that

$$
\max \left\{s_{\tau(n)}, s_{n}\right\} \leq s_{\tau(n)+1}, \forall n \geq n_{0} .
$$

From (3.8), we have

$$
0 \leq \sigma_{\tau(n)} \leq b_{\tau(n)}\left(c_{\tau(n)}-s_{\tau(n)}\right) \rightarrow 0 .
$$

Thus $\sigma_{\tau(n)} \rightarrow 0$. By similar argument to Case 1 , we get

$$
\limsup _{n \rightarrow \infty}\left\langle F(p), j\left(p-x_{\tau(n)+1}\right)\right\rangle \leq 0,
$$

or $\lim \sup _{n \rightarrow \infty} c_{\tau(n)} \leq 0$. From $s_{\tau(n)}<s_{\tau(n+1)}, b_{n}>0, \sigma_{n} \geq 0$ and the following estimate

$$
s_{\tau(n+1)} \leq\left(1-b_{\tau(n)}\right) s_{\tau(n)}+b_{\tau(n)} c_{\tau(n)}-\sigma_{\tau(n)}
$$


we obtain $s_{\tau(n)} \leq c_{\tau(n)}$. Hence, it follows from $\lim \sup _{n \rightarrow \infty} c_{\tau(n)} \leq 0$ that $\lim \sup _{n \rightarrow \infty} s_{\tau(n)} \leq 0$. Thus

$$
\lim _{n \rightarrow \infty} s_{\tau(n)}=0 .
$$

Similar to (3.10), we have

$$
\left\|x_{\tau(n)+1}-x_{\tau(n)}\right\| \rightarrow 0
$$

Thus, from the boundedness of $\left\{x_{n}\right\}$, we get

$$
\begin{aligned}
\left|s_{\tau(n)+1}-s_{\tau(n)}\right| & =\left|\left\|x_{\tau(n)+1}-p\right\|^{2}-\left\|x_{\tau(n)+1}-p\right\|^{2}\right| \\
& \leq\left\|x_{\tau(n)+1}-x_{\tau(n)}\right\|\left(\left\|x_{\tau(n)+1}-p\right\|+\left\|x_{\tau(n)+1}-p\right\|\right) \rightarrow 0 .
\end{aligned}
$$

Hence, $\left|s_{\tau(n)+1}-s_{\tau(n)}\right| \rightarrow 0$. From (3.12) and (3.14), for all $n \geq n_{0}$, we have

$$
0 \leq s_{n} \leq s_{\tau(n)+1}=s_{\tau(n)}+\left(s_{\tau(n)+1}-s_{\tau(n)}\right) \rightarrow 0,
$$

which implies that $s_{n} \rightarrow 0$. Consequently, we obtain $s_{n} \rightarrow 0$ in both cases, that is, $x_{n} \rightarrow p$.

This completes the proof.

In the case that $N=1$, we have the following corollary:

Corollary 3.1. Let $E$ be a real uniformly convex Banach space with a uniformly Gâteaux differential norm. Assume that $F: E \longrightarrow E$ is $\delta$-strongly accretive and $\lambda$-strictly pseudocontractive with $\delta+\lambda>1$. Let $A: E \longrightarrow 2^{E}$ be an m-accretive operator such that $S=A^{-1} 0 \neq \emptyset$. If the sequences $\left\{\lambda_{n}\right\},\left\{r_{n}\right\}$ and $\left\{\beta_{n}\right\}$ satisfy the following conditions:

i) $\inf _{n}\left\{r_{n}\right\} \geq r>0$;

ii) $\left\{\beta_{n}\right\} \subset(\alpha, \beta)$ with $\alpha, \beta \in(0,1)$ for all $i=1,2, \ldots, N$;

iii) $\left\{\lambda_{n}\right\} \subset(0,1), \lim _{n \rightarrow \infty} \lambda_{n}=0, \sum_{n=0}^{\infty} \lambda_{n}=\infty$,

then the sequence $\left\{x_{n}\right\}$ defined by $x_{0} \in E$ and

$$
\left\{\begin{array}{l}
y_{n}=\left(1-\beta_{n}\right) x_{n}+\beta_{n} J_{r_{n}}^{A} x_{n}, \\
x_{n+1}=\left(I-\lambda_{n} F\right)\left(y_{n}\right), n \geq 0
\end{array}\right.
$$

converges strongly to an element $p \in S$, which is the unique solution of $\mathrm{VI}^{*}(F, S)$.

Remark 3.1. Corollary 3.1 is more general than Theorem 5.7 in [5].

Next, we give an analogue result in the case $A_{i}$ is maximal monotone operators in a real Hilbert space $H$ and $F$ is $L$-Lipschitz and $\eta$-strongly monotone operator. We need the following lemma.

Lemma 3.1. [18] Let $F: H \longrightarrow H$ be an L-Lipschitz and $\eta$-strongly monotone operator. Then $f=$ $I-\lambda \mu F$ is a contraction mapping with the contraction coefficient $c=1-\sqrt{1-\mu\left(2 \eta-\mu L^{2}\right)}$, for each $\mu \in\left(0,2 \eta / L^{2}\right)$ and $\lambda \in[0,1]$.

So, by using Lemma 3.1 and by a similar argument to the proof of Theorem 3.1, we get the following theorem.

Theorem 3.2. Let $H$ be a real Hilbert space. Assume that $F: H \longrightarrow H$ is an L-Lipschitz and $\eta$ strongly monotone operator. Let $A_{i}: H \longrightarrow 2^{H}, i=1,2, \ldots, N$, be maximal monotone operators such that $S=\cap_{i=1}^{N} A_{i}^{-1} 0 \neq \emptyset$. If the sequences $\left\{\lambda_{n}\right\},\left\{r_{n}^{i}\right\}$, and $\left\{\beta_{n}^{i}\right\}, i=1,2, \ldots, N$ satisfy the following conditions:

i) $\min _{i=1,2, \ldots, N}\left\{\inf _{n}\left\{r_{n}^{i}\right\}\right\} \geq r>0$ for all $i=1,2, \ldots, N$; 
ii) $\left\{\beta_{n}^{i}\right\} \subset(\alpha, \beta)$ with $\alpha, \beta \in(0,1)$ for all $i=1,2, \ldots, N$;

iii) $\left\{\lambda_{n}\right\} \subset(0,1), \lim _{n \rightarrow \infty} \lambda_{n}=0, \sum_{n=0}^{\infty} \lambda_{n}=\infty$,

then, for any $\mu \in\left(0,2 \eta / L^{2}\right)$, the sequence $\left\{x_{n}\right\}$ defined by $x_{0} \in H$ and

$$
\begin{aligned}
& y_{n}^{0}=x_{n}, n \geq 0, \\
& y_{n}^{i}=\left(1-\beta_{n}^{i}\right) y_{n}^{i-1}+\beta_{n}^{i} J_{i, n} y_{n}^{i-1}, i=1,2, \ldots, N, n \geq 0, J_{i, n}=J_{r_{n}^{i}}^{A_{i}}, \\
& x_{n+1}=\left(I-\lambda_{n} \mu F\right)\left(y_{n}^{N}\right), n \geq 0
\end{aligned}
$$

converges strongly to an element $p \in S$, which is the unique solution of $\operatorname{VI}(F, S)$.

3.2. A parallel algorithm. In this section, we introduce a new parallel algorithm for solving Problem (3.1).

Algorithm 3.2. For any $x_{0} \in E$, we define the sequence $\left\{x_{n}\right\}$ by

$$
\begin{aligned}
& y_{n}^{i}=\left(1-\beta_{n}^{i}\right) x_{n}+\beta_{n}^{i} J_{i, n} x_{n}, i=1,2, \ldots, N, n \geq 0, J_{i, n}=J_{r_{n}^{i}}^{A_{i}}, \\
& \text { chosse } i_{n} \text { such that }\left\|y_{n}^{i_{n}}-x_{n}\right\|=\max _{i=1, \ldots, N}\left\{\left\|y_{n}^{i}-x_{n}\right\|\right\}, \text { let } y_{n}=y_{n}^{i_{n}}, \\
& x_{n+1}=\left(I-\lambda_{n} F\right)\left(y_{n}\right), n \geq 0,
\end{aligned}
$$

where $\left\{\lambda_{n}\right\},\left\{r_{n}^{i}\right\}$, and $\left\{\beta_{n}^{i}\right\}, i=1,2, \ldots, N$, are sequences of positive real numbers.

The strong convergence of Algorithm 3.2 is given by the following theorem.

Theorem 3.3. Let $\left\{x_{n}\right\}$ be a sequence generated by Algorithm 3.2. If the sequences $\left\{\lambda_{n}\right\}$, $\left\{r_{n}^{i}\right\}$, and $\left\{\beta_{n}^{i}\right\}, i=1,2, \ldots, N$ satisfy the following conditions:

i) $\min _{i=1,2, \ldots, N}\left\{\inf _{n}\left\{r_{n}^{i}\right\}\right\} \geq r>0$ for all $i=1,2, \ldots, N$;

ii) $\left\{\beta_{n}^{i}\right\} \subset(\alpha, \beta)$ with $\alpha, \beta \in(0,1)$ for all $i=1,2, \ldots, N$;

iii) $\left\{\lambda_{n}\right\} \subset(0,1), \lim _{n \rightarrow \infty} \lambda_{n}=0, \sum_{n=0}^{\infty} \lambda_{n}=\infty$,

then the sequence $\left\{x_{n}\right\}$ converges strongly to an element $p \in S$, which is the unique solution of $\mathrm{VI}^{*}(F, S)$.

Proof. First, we show that $\left\{x_{n}\right\}$ is bounded. Indeed, letting $u \in S$, we have

$$
\begin{aligned}
\left\|y_{n}^{i}-u\right\| & =\left\|\left(1-\beta_{n}^{i}\right) x_{n}+\beta_{n}^{i} J_{i, n} x_{n}-u\right\| \\
& \leq\left(1-\beta_{n}^{i}\right)\left\|x_{n}-u\right\|+\beta_{n}^{i}\left\|J_{i, n} x_{n}-J_{i, n} u\right\| \\
& \leq\left(1-\beta_{n}^{i}\right)\left\|x_{n}-u\right\|+\beta_{n}^{i}\left\|x_{n}-u\right\| \\
& \leq\left\|x_{n}-u\right\|,
\end{aligned}
$$

for all $i=1,2, \ldots, N$. From Lemma 2.2, we have

$$
\begin{aligned}
\left\|x_{n+1}-u\right\| & =\left\|\left(I-\lambda_{n} F\right)\left(y_{n}\right)-u\right\| \\
& =\left\|\left(I-\lambda_{n} F\right)\left(y_{n}\right)-\left(I-\lambda_{n} F\right)(u)-\lambda_{n} F(u)\right\| \\
& \leq\left\|\left(I-\lambda_{n} F\right)\left(y_{n}\right)-\left(I-\lambda_{n} F\right)(u)\right\|+\lambda_{n}\|F(u)\| \\
& \leq\left(1-\lambda_{n}\left(1-\sqrt{\frac{1-\delta}{\lambda}}\right)\right)\left\|y_{n}-u\right\|+\lambda_{n}\|F(u)\| \\
& \leq \max \left\{\left\|y_{n}-u\right\|,\left(1-\sqrt{\frac{1-\delta}{\lambda}}\right)^{-1}\|F(u)\|\right\} .
\end{aligned}
$$


From (3.18), (3.19), and the definition of $y_{n}$, we get

$$
\left\|x_{n+1}-u\right\| \leq \max \left\{\left\|x_{n}-u\right\|,\left(1-\sqrt{\frac{1-\delta}{\lambda}}\right)^{-1}\|F(u)\|\right\}
$$

By induction, we obtain

$$
\left\|x_{n}-u\right\| \leq \max \left\{\left\|x_{0}-u\right\|,\left(1-\sqrt{\frac{1-\delta}{\lambda}}\right)^{-1}\|F(u)\|\right\}
$$

Thus, $\left\{x_{n}\right\}$ is bounded. So $\left\{y_{n}^{i}\right\},\left\{F\left(y_{n}^{i}\right)\right\}, i=1,2, \ldots, N$ are also bounded. Let $p$ is the unique solution of $\operatorname{VI}^{*}(F, S)$, that is,

$$
\langle F(p), j(p-u)\rangle \leq 0, \forall u \in S,
$$

From (3.2), we have

$$
\begin{aligned}
\left\|x_{n+1}-p\right\|^{2}= & \left\langle\left(I-\lambda_{n} F\right)\left(y_{n}\right)-p, j\left(x_{n+1}-p\right)\right\rangle \\
= & \left\langle\left(I-\lambda_{n} F\right)\left(y_{n}\right)-\left(I-\lambda_{n} F\right)(p), j\left(x_{n+1}-p\right)\right\rangle \\
& +\lambda_{n}\left\langle F(p), j\left(p-x_{n+1}\right)\right\rangle \\
\leq & \left(1-\lambda_{n}\left(1-\sqrt{\frac{1-\delta}{\lambda}}\right)\right)\left\|y_{n}-p\right\| \cdot\left\|x_{n+1}-p\right\| \\
& +\lambda_{n}\left\langle F(p), j\left(p-x_{n+1}\right)\right\rangle \\
\leq & \left(1-\lambda_{n}\left(1-\sqrt{\frac{1-\delta}{\lambda}}\right)\right) \frac{\left\|y_{n}-p\right\|^{2}+\left\|x_{n+1}-p\right\|^{2}}{2} \\
& +\lambda_{n}\left\langle F(p), j\left(p-x_{n+1}\right)\right\rangle .
\end{aligned}
$$

This implies that

$$
\begin{aligned}
\left\|x_{n+1}-p\right\|^{2} \leq & \frac{\left(1-\lambda_{n}\left(1-\sqrt{\frac{1-\delta}{\lambda}}\right)\right)}{1+\lambda_{n}\left(1-\sqrt{\frac{1-\delta}{\lambda}}\right)}\left\|y_{n}-p\right\|^{2} \\
& +\frac{2 \lambda_{n}}{1+\lambda_{n}\left(1-\sqrt{\frac{1-\delta}{\lambda}}\right)}\left\langle F(p), j\left(p-x_{n+1}\right)\right\rangle \\
= & \left(1-\frac{2 \lambda_{n}\left(1-\sqrt{\frac{1-\delta}{\lambda}}\right)}{1+\lambda_{n}\left(1-\sqrt{\frac{1-\delta}{\lambda}}\right)}\right)\left\|y_{n}-p\right\|^{2} \\
& +\frac{2 \lambda_{n}\left(1-\sqrt{\frac{1-\delta}{\lambda}}\right)}{1+\lambda_{n}\left(1-\sqrt{\frac{1-\delta}{\lambda}}\right)}\left(1-\sqrt{\frac{1-\delta}{\lambda}}\right)^{-1}\left\langle F(p), j\left(p-x_{n+1}\right)\right\rangle .
\end{aligned}
$$


From Lemma 2.1, we have

$$
\begin{aligned}
\left\|y_{n}-p\right\|^{2}= & \left\|\left(1-\beta_{n}^{i_{n}}\right) x_{n}+\beta_{n}^{i_{n}} J_{i_{n}, n} x_{n}-p\right\|^{2} \\
\leq & \left(1-\beta_{n}^{i_{n}}\right)\left\|x_{n}-p\right\|^{2}+\beta_{n}^{i_{n}}\left\|J_{i_{n}, n} x_{n}-p\right\|^{2} \\
& -\beta_{n}^{i_{n}}\left(1-\beta_{n}^{i_{n}}\right) \varphi\left(\left\|x_{n}-J_{i_{n}, n} x_{n}\right\|\right) .
\end{aligned}
$$

From (3.21) and (3.22), we obtain

$$
\begin{aligned}
\left\|x_{n+1}-p\right\|^{2} \leq & \left(1-\frac{2 \lambda_{n}\left(1-\sqrt{\frac{1-\delta}{\lambda}}\right)}{1+\lambda_{n}\left(1-\sqrt{\frac{1-\delta}{\lambda}}\right)}\right)\left\|x_{n}-p\right\|^{2} \\
& +\frac{2 \lambda_{n}\left(1-\sqrt{\frac{1-\delta}{\lambda}}\right)}{1+\lambda_{n}\left(1-\sqrt{\frac{1-\delta}{\lambda}}\right)}\left(1-\sqrt{\frac{1-\delta}{\lambda}}\right)^{-1}\left\langle F(p), j\left(p-x_{n+1}\right)\right\rangle \\
& -\alpha(1-\beta)\left(1-\frac{2 \lambda_{n}\left(1-\sqrt{\frac{1-\delta}{\lambda}}\right)}{1+\lambda_{n}\left(1-\sqrt{\frac{1-\delta}{\lambda}}\right)}\right) \varphi\left(\left\|x_{n}-J_{i_{n}, n} x_{n}\right\|\right) .
\end{aligned}
$$

Putting

and

$$
\begin{gathered}
s_{n}=\left\|x_{n}-p\right\|^{2}, \\
b_{n}=\frac{2 \lambda_{n}\left(1-\sqrt{\frac{1-\delta}{\lambda}}\right)}{1+\lambda_{n}\left(1-\sqrt{\frac{1-\delta}{\lambda}}\right)},
\end{gathered}
$$

$$
\begin{gathered}
c_{n}=\left(1-\sqrt{\frac{1-\delta}{\lambda}}\right)^{-1}\left\langle F(p), j\left(p-x_{n+1}\right)\right\rangle, \\
\left.\sigma_{n}=\left(1-b_{n}\right) \alpha(1-\beta)\right) \varphi\left(\left\|x_{n}-J_{i_{n}, n} x_{n}\right\|\right) .
\end{gathered}
$$

we obtain that inequality (3.23) can be rewritten as

$$
s_{n+1} \leq\left(1-b_{n}\right) s_{n}+b_{n} c_{n}-\sigma_{n} .
$$

We will show that $s_{n} \rightarrow 0$ by considering two possible cases.

Case 1. $\left\{s_{n}\right\}$ is eventually decreasing, i.e., there exists $N_{0} \geq 0$ such that $\left\{s_{n}\right\}$ is decreasing for $n \geq N_{0}$ and thus $\left\{s_{n}\right\}$ must be convergent. It then follows from (3.8) that

$$
0 \leq \sigma_{n} \leq\left(s_{n}-s_{n+1}\right)+b_{n}\left(c_{n}-s_{n}\right) \rightarrow 0,
$$

which implies that

$$
\left\|x_{n}-J_{i_{n}, n} x_{n}\right\| \rightarrow 0 .
$$

Thus, we have

$$
\left\|y_{n}-x_{n}\right\|=\beta_{n}^{i_{n}}\left\|x_{n}-J_{i_{n}, n} x_{n}\right\| \rightarrow 0 .
$$

From the definition of $y_{n}$, we get

$$
\left\|y_{n}^{i}-x_{n}\right\| \rightarrow 0
$$


for all $i=1,2, \ldots, N$, Hence

$$
\left\|x_{n}-J_{i, n} x_{n}\right\|=\frac{1}{\beta_{n}^{i}}\left\|y_{n}^{i}-x_{n}\right\| \rightarrow 0
$$

for all $i=1,2, \ldots, N$. Let $T=\frac{1}{N} \sum_{i=1}^{N} J_{r}^{A_{i}}$. Then $T$ is a nonexpansive mapping and $S=\operatorname{Fix}(T)$. From $\left\|x_{n}-T_{i}\left(x_{n}\right)\right\| \rightarrow 0$ for all $i=1,2, \ldots, N$ and by the following estimate

$$
\left\|x_{n}-T\left(x_{n}\right)\right\| \leq \frac{1}{N} \sum_{i=1}^{N}\left\|x_{n}-T_{i}\left(x_{n}\right)\right\|,
$$

we get $\lim _{n \rightarrow \infty}\left\|x_{n}-T\left(x_{n}\right)\right\|=0$. From Proposition 1.1 and Proposition 1.2, we obtain

$$
\limsup _{n \rightarrow \infty}\left\langle F(p), j\left(p-x_{n}\right)\right\rangle \leq 0 .
$$

Letting $K=\sup _{n}\left\{\left\|F\left(y_{n}\right)\right\|\right\}$, from (3.25) and Lemma 2.2, we have

$$
\begin{aligned}
\left\|x_{n+1}-x_{n}\right\| & =\left\|\left(I-\lambda_{n} F\right)\left(y_{n}\right)-x_{n}\right\| \\
& \leq\left\|\left(I-\lambda_{n} F\right)\left(x_{n}\right)-\left(I-\lambda_{n} F\right)\left(y_{n}\right)\right\|+\lambda_{n} K \\
& \leq\left(1-\lambda_{n}\left(1-\sqrt{\frac{1-\delta}{\lambda}}\right)\right)\left\|x_{n}-y_{n}\right\|+\lambda_{n} K \rightarrow 0,
\end{aligned}
$$

which implies that

$$
\left\|x_{n+1}-x_{n}\right\| \rightarrow 0
$$

Thus, by (3.27) and the fact that the duality map $j$ is uniformly norm-to-weak* continuous on bounded set, we get

$$
\limsup _{n \rightarrow \infty}\left\langle F(p), j\left(p-x_{n+1}\right)\right\rangle \leq 0,
$$

that is, $\limsup _{n \rightarrow \infty} c_{n} \leq 0$. From (3.24), we have

$$
s_{n+1} \leq\left(1-b_{n}\right) s_{n}+b_{n} c_{n} .
$$

Applying Lemma 2.6, we obtain $\lim _{n \rightarrow \infty} s_{n}=0$.

Case 2. $\left\{s_{n}\right\}$ is not eventually decreasing. Hence, there exists a subsequence $\left\{s_{n_{k}}\right\}$ of $\left\{s_{n}\right\}$ such that $s_{n_{k}} \leq s_{n_{k}+1}$ for all $k \geq 0$. By Lemma 2.3, we can define a subsequence $\left\{s_{\tau(n)}\right\}$ such that

$$
\max \left\{s_{\tau(n)}, s_{n}\right\} \leq s_{\tau(n)+1}, \forall n \geq n_{0} .
$$

From (3.24), we have

$$
0 \leq \sigma_{\tau(n)} \leq b_{\tau(n)}\left(c_{\tau(n)}-s_{\tau(n)}\right) \rightarrow 0 .
$$

Thus $\sigma_{\tau(n)} \rightarrow 0$. By a similar argument to Case 1 , we get

$$
\limsup _{n \rightarrow \infty}\left\langle F(p), j\left(p-x_{\tau(n)+1}\right)\right\rangle \leq 0,
$$

or $\lim \sup _{n \rightarrow \infty} c_{\tau(n)} \leq 0$. From $s_{\tau(n)}<s_{\tau(n+1)}, b_{n}>0, \sigma_{n} \geq 0$ and the following estimate

$$
s_{\tau(n+1)} \leq\left(1-b_{\tau(n)}\right) s_{\tau(n)}+b_{\tau(n)} c_{\tau(n)}-\sigma_{\tau(n)},
$$


we obtain $s_{\tau(n)} \leq c_{\tau(n)}$. Hence, it follows from $\lim \sup _{n \rightarrow \infty} c_{\tau(n)} \leq 0$ that $\lim \sup _{n \rightarrow \infty} s_{\tau(n)} \leq 0$. Thus

$$
\lim _{n \rightarrow \infty} s_{\tau(n)}=0 .
$$

Similar to (3.28), we have

$$
\left\|x_{\tau(n)+1}-x_{\tau(n)}\right\| \rightarrow 0 .
$$

Thus, from the boundedness of the sequence $\left\{x_{n}\right\}$, we get

$$
\begin{aligned}
\left|s_{\tau(n)+1}-s_{\tau(n)}\right| & =\left|\left\|x_{\tau(n)+1}-p\right\|^{2}-\left\|x_{\tau(n)+1}-p\right\|^{2}\right| \\
& \leq\left\|x_{\tau(n)+1}-x_{\tau(n)}\right\|\left(\left\|x_{\tau(n)+1}-p\right\|+\left\|x_{\tau(n)+1}-p\right\|\right) \rightarrow 0 .
\end{aligned}
$$

Hence, $\left|s_{\tau(n)+1}-s_{\tau(n)}\right| \rightarrow 0$. From (3.30) and (3.32), for all $n \geq n_{0}$, we have

$$
0 \leq s_{n} \leq s_{\tau(n)+1}=s_{\tau(n)}+\left(s_{\tau(n)+1}-s_{\tau(n)}\right) \rightarrow 0,
$$

which implies that $s_{n} \rightarrow 0$. Consequently, we obtain $s_{n} \rightarrow 0$ in both cases, that is, $x_{n} \rightarrow p$. This completes the proof.

So, by using Lemma 3.1 and by a similar argument to the proof of Theorem 3.3, we get the following theorem.

Theorem 3.4. Let $H$ be a real Hilbert space. Assume that $F: H \longrightarrow H$ is an L-Lipschitz and $\eta$ strongly monotone operator. Let $A_{i}: H \longrightarrow 2^{H}, i=1,2, \ldots, N$, be maximal monotone operators such that $S=\cap_{i=1}^{N} A_{i}^{-1} 0 \neq \emptyset$. If the sequences $\left\{\lambda_{n}\right\},\left\{r_{n}^{i}\right\}$, and $\left\{\beta_{n}^{i}\right\}, i=1,2, \ldots, N$ satisfy the following conditions:

i) $\min _{i=1,2, \ldots, N}\left\{\inf _{n}\left\{r_{n}^{i}\right\}\right\} \geq r>0$ for all $i=1,2, \ldots, N$;

ii) $\left\{\beta_{n}^{i}\right\} \subset(\alpha, \beta)$ with $\alpha, \beta \in(0,1)$ for all $i=1,2, \ldots, N$;

iii) $\left\{\lambda_{n}\right\} \subset(0,1), \lim _{n \rightarrow \infty} \lambda_{n}=0, \sum_{n=0}^{\infty} \lambda_{n}=\infty$,

then, for any $\mu \in\left(0,2 \eta / L^{2}\right)$, the sequence $\left\{x_{n}\right\}$ defined by $x_{0} \in H$ and

$$
\begin{aligned}
& y_{n}^{i}=\left(1-\beta_{n}^{i}\right) x_{n}+\beta_{n}^{i} J_{i, n} x_{n}, i=1,2, \ldots, N, n \geq 0, J_{i, n}=J_{r_{n}^{i}}^{A_{i}}, \\
& \text { chosse } i_{n} \text { such that }\left\|y_{n}^{i_{n}}-x_{n}\right\|=\max _{i=1, \ldots, N}\left\{\left\|y_{n}^{i}-x_{n}\right\|\right\}, \text { let } y_{n}=y_{n}^{i_{n}}, \\
& x_{n+1}=\left(I-\lambda_{n} \mu F\right)\left(y_{n}\right), n \geq 0
\end{aligned}
$$

converges strongly to an element $p \in S$, which is the unique solution of $\operatorname{VI}(F, S)$.

\section{Applications}

By the careful analysis of the proof of Theorem 3.1 and Theorem 3.3, we can obtain the following result for the problem of finding a common fixed point of a family of finite nonexpansive mappings in a uniformly convex Banach space.

Theorem 4.1. Let $E$ be a real uniformly convex Banach space with a uniformly Gâteaux differential norm. Assume that $F: E \longrightarrow E$ is $\delta$-strongly accretive and $\lambda$-strictly pseudocontractive with $\delta+\lambda>$ 1. Let $T_{i}: E \longrightarrow E, i=1,2, \ldots, N$, be nonexpansive mappings such that $S=\cap_{i=1}^{N} \operatorname{Fix}\left(T_{i}\right) \neq \emptyset$. If the sequences $\left\{\lambda_{n}\right\}$ and $\left\{\beta_{n}^{i}\right\}, i=1,2, \ldots, N$ satisfy the following conditions:

i) $\left\{\beta_{n}^{i}\right\} \subset(\alpha, \beta)$ with $\alpha, \beta \in(0,1)$ for all $i=1,2, \ldots, N$;

ii) $\left\{\lambda_{n}\right\} \subset(0,1), \lim _{n \rightarrow \infty} \lambda_{n}=0, \sum_{n=0}^{\infty} \lambda_{n}=\infty$, 
then the sequence $\left\{x_{n}\right\}$ defined by $x_{0} \in E$ and

$$
\begin{aligned}
& y_{n}^{0}=x_{n}, n \geq 0, \\
& y_{n}^{i}=\left(1-\beta_{n}^{i}\right) y_{n}^{i-1}+\beta_{n}^{i} T_{i} y_{n}^{i-1}, i=1,2, \ldots, N, n \geq 0, \\
& x_{n+1}=\left(I-\lambda_{n} F\right)\left(y_{n}^{N}\right), n \geq 0,
\end{aligned}
$$

or

$$
\begin{aligned}
& y_{n}^{i}=\left(1-\beta_{n}^{i}\right) x_{n}+\beta_{n}^{i} T_{i} x_{n}, i=1,2, \ldots, N, n \geq 0, \\
& \text { chosse } i_{n} \text { such that }\left\|y_{n}^{i_{n}}-x_{n}\right\|=\max _{i=1, \ldots, N}\left\{\left\|y_{n}^{i}-x_{n}\right\|\right\}, \text { let } y_{n}=y_{n}^{i_{n}}, \\
& x_{n+1}=\left(I-\lambda_{n} F\right)\left(y_{n}\right), n \geq 0,
\end{aligned}
$$

converges strongly to an element $p \in S$, which is a unique solution of $\mathrm{VI}^{*}(F, S)$.

Remark 4.1. Theorem 4.1 is more general than the result of Yamada [18] (Theorem 3.3), it does not require the conditions:

$$
C=\operatorname{Fix}\left(T_{N} \ldots T_{1}\right)=\operatorname{Fix}\left(T_{1} T_{N} \ldots T_{2}\right)=\ldots=\operatorname{Fix}\left(T_{N-1} \ldots T_{1} T_{N}\right),
$$

and $\sum_{n=1}^{\infty}\left|\lambda_{n+N}-\lambda_{n}\right|<\infty$. Moreover, iterative method (4.2) is a new result for solving the variational inequality over the set of common fixed points of a finite family of nonexpansive mappings in Banach spaces.

Let $H$ be a real Hilbert space. We consider variational inequality (1.1) with the fact thats $F: H \longrightarrow H$ is $L$-Lipschitz and $\eta$-strongly monotone operator and $C=\cap_{i=1}^{N} C_{i}$, where $C_{i}$ is a nonempty closed convex subset of $H$. Let $T_{i}=P_{C_{i}}$, where $P_{C_{i}}$ is metric projection from $H$ onto $C_{i}$ for all $i=1,2, \ldots, N$. By the careful analysis of Theorem 3.2, we obtain the following theorem.

Theorem 4.2. If the sequences $\left\{\lambda_{n}\right\}$ and $\left\{\beta_{n}^{i}\right\}, i=1,2, \ldots, N$ satisfy the following conditions:

i) $\left\{\beta_{n}^{i}\right\} \subset(\alpha, \beta)$ with $\alpha, \beta \in(0,1)$ for all $i=1,2, \ldots, N$;

ii) $\left\{\lambda_{n}\right\} \subset(0,1), \lim _{n \rightarrow \infty} \lambda_{n}=0, \sum_{n=0}^{\infty} \lambda_{n}=\infty$,

then, for any $\mu \in\left(0,2 \eta / k^{2}\right)$, the sequence $\left\{x_{n}\right\}$ defined by $x_{0} \in H$ and

$$
\begin{aligned}
& y_{n}^{0}=x_{n}, n \geq 0, \\
& y_{n}^{i}=\left(1-\beta_{n}^{i}\right) y_{n}^{i-1}+\beta_{n}^{i} P_{C_{i}} y_{n}^{i-1}, i=1,2, \ldots, N, n \geq 0, \\
& x_{n+1}=\left(I-\lambda_{n} \mu F\right)\left(y_{n}^{N}\right), n \geq 0
\end{aligned}
$$

or

$$
\begin{aligned}
& y_{n}^{i}=\left(1-\beta_{n}^{i}\right) x_{n}+\beta_{n}^{i} P_{C_{i}} x_{n}, i=1,2, \ldots, N, n \geq 0, \\
& \text { chosse } i_{n} \text { such that }\left\|y_{n}^{i_{n}}-x_{n}\right\|=\max _{i=1, \ldots, N}\left\{\left\|y_{n}^{i}-x_{n}\right\|\right\}, \text { let } y_{n}=y_{n}^{i_{n}}, \\
& x_{n+1}=\left(I-\lambda_{n} \mu F\right)\left(y_{n}\right), n \geq 0
\end{aligned}
$$

converges strongly to an element $p \in C$, which is the unique solution of $\operatorname{VI}(F, C)$.

We have the following corollary for the convex feasibility problem.

Corollary 4.1. Let $C_{i}$ be a nonempty closed convex subset of a real Hilbert space $H, i=1,2, \ldots, N$, with $C=\cap_{i=1}^{N} C_{i} \neq \emptyset$. If the sequences $\left\{\lambda_{n}\right\}$ and $\left\{\beta_{n}^{i}\right\}, i=1,2, \ldots, N$ satisfy the following conditions: 
i) $\left\{\beta_{n}^{i}\right\} \subset(\alpha, \beta)$ with $\alpha, \beta \in(0,1)$ for all $i=1,2, \ldots, N$;

ii) $\left\{\lambda_{n}\right\} \subset(0,1), \lim _{n \rightarrow \infty} \lambda_{n}=0, \sum_{n=0}^{\infty} \lambda_{n}=\infty$,

then the sequence $\left\{x_{n}\right\}$ defined by $u, x_{0} \in H$ and

$$
\begin{aligned}
& y_{n}^{0}=x_{n}, n \geq 0, \\
& y_{n}^{i}=\left(1-\beta_{n}^{i}\right) y_{n}^{i-1}+\beta_{n}^{i} P_{C_{i}} y_{n}^{i-1}, i=1,2, \ldots, N, n \geq 0, \\
& x_{n+1}=\lambda_{n} u+\left(1-\lambda_{n}\right) y_{n}^{N}, n \geq 0
\end{aligned}
$$

or

$$
\begin{aligned}
& y_{n}^{i}=\left(1-\beta_{n}^{i}\right) x_{n}+\beta_{n}^{i} P_{C_{i}} x_{n}, i=1,2, \ldots, N, n \geq 0, \\
& \text { chosse } i_{n} \text { such that }\left\|y_{n}^{i_{n}}-x_{n}\right\|=\max _{i=1, \ldots, N}\left\{\left\|y_{n}^{i}-x_{n}\right\|\right\}, \text { let } y_{n}=y_{n}^{i_{n}}, \\
& x_{n+1}=\lambda_{n} u+\left(1-\lambda_{n}\right) y_{n}, n \geq 0
\end{aligned}
$$

converges strongly to an element $P_{C} u \in C$, where $P_{C}: H \longrightarrow C$ is the metric projection from $H$ onto $C$.

Proof. Let $f(x)=\frac{1}{2}\|x-u\|^{2}$ for all $x \in H$. Then $F=\nabla f=x-u$, for all $x \in H$, is a 1-Lipschitz and 1-strongly monotone operator in $H$. So, applying Theorem 4.2 with $\mu=1$, we get the proof of this corollary. This completes the proof.

\section{A NUMERICAL EXAMPLE}

Example 5.1. Consider the problem of finding an element $x^{*} \in S$ such that

$$
\varphi\left(x^{*}\right)=\min _{x \in S} \varphi(x),
$$

where $\varphi(x)=\left(x_{1}+1\right)^{2}+\left(x_{2}-1\right)^{2}+x_{3}^{2}$ for all $x=\left(x_{1}, x_{2}, x_{3}\right) \in \mathbb{R}^{3}, S=\cap_{i=1}^{100} C_{i}$ with

$$
C_{i}=\left\{\left(x_{1}, x_{2}, x_{3}\right):\left(x_{1}+1 / i\right)^{2}+\left(x_{2}-1 / i\right)^{2}+x_{3}^{2} \leq 2\right\}, i=1,2, \ldots, 100 .
$$

It is easy to show that $\varphi$ is a convex function for $F=\nabla \varphi$ is a 2-Lipschitz, 2-strongly monotone operator, and $x^{*}=(-1,1,0)$ is the minimum point of $\varphi$ on $S$.

a) Numerical results for Algorithm 3.1

- Applying iterative process (4.3) with $\mu=9 / 10, \beta_{n}^{i}=1 / 2$ and $\lambda_{n}=1 / n$ for all $n \geq 1$ and for all $i=1,2, \ldots, N$, and $x^{0}=(3,4,5)$, we obtain the following table of results:

\begin{tabular}{llll}
\hline TOL & $\left\|x^{n}-x^{*}\right\|$ & $n$ & $x^{n}$ \\
\hline \hline $10^{-5}$ & $9.96 \times 10^{-6}$ & 193 & $\left(-1.000003,9.99993 \times 10^{-1},-6.95 \times 10^{-6}\right)$ \\
$10^{-6}$ & $9.97 \times 10^{-7}$ & 692 & $\left(-1.000000,9.99999 \times 10^{-1},-6.96 \times 10^{-7}\right)$ \\
$10^{-7}$ & $9.99 \times 10^{-8}$ & 2483 & $\left(-1.000000,9.99999 \times 10^{-1},-6.98 \times 10^{-8}\right)$ \\
$10^{-8}$ & $9.99 \times 10^{-9}$ & 8922 & $\left(-1.000000,9.99999 \times 10^{-1},-6.98 \times 10^{-9}\right)$ \\
$10^{-9}$ & $9.99 \times 10^{-10}$ & 32063 & $\left(-1.000000,9.99999 \times 10^{-1},-6.98 \times 10^{-10}\right)$ \\
\hline
\end{tabular}

TABLE 1. Table of numerical results

- Applying iterative process (4.3) with $\mu=9 / 10, \beta_{n}^{i}=1 / 2+1 / 4 \sqrt{n}$ and $\lambda_{n}=1 / \sqrt{n}$ for all $n \geq 1$ and for all $i=1,2, \ldots, N$ (note that in this case the sequences $\left\{\beta_{n}^{i}\right\}$ and $\left\{\lambda_{n}\right\}$ do not satisfy the conditions 


\begin{tabular}{llll}
\hline TOL & $\left\|x^{n}-x^{*}\right\|$ & $n$ & $x^{n}$ \\
\hline \hline $10^{-5}$ & $3.69 \times 10^{-6}$ & 8 & $\left(-1.000001,9.99998 \times 10^{-1},-2.62 \times 10^{-6}\right)$ \\
$10^{-6}$ & $6.37 \times 10^{-7}$ & 10 & $\left(-1.000000,9.99999 \times 10^{-1},-4.52 \times 10^{-7}\right)$ \\
$10^{-7}$ & $7.00 \times 10^{-8}$ & 13 & $\left(-1.000000,9.99999 \times 10^{-1},-4.97 \times 10^{-8}\right)$ \\
$10^{-8}$ & $6.03 \times 10^{-9}$ & 17 & $\left(-1.000000,9.99999 \times 10^{-1},-4.28 \times 10^{-9}\right)$ \\
$10^{-9}$ & $7.39 \times 10^{-10}$ & 21 & $\left(-1.000000,9.99999 \times 10^{-1},-5.25 \times 10^{-10}\right)$ \\
\hline \multicolumn{4}{c}{ TABLE 2. Table of numerical results }
\end{tabular}

$\sum_{n=1}^{\infty}\left|\beta_{n+1}^{i}-\beta_{n}^{i}\right|<\infty$ for all $i=1,2, \ldots, N$ and $\left.\sum_{n=1}^{\infty}\left|\lambda_{n+1}-\lambda_{n}\right|<\infty\right)$, and $x^{0}=(3,4,5)$, we obtain the following table of results:

The strong convergence of iterative process (4.3) is also described in Fig. 1.

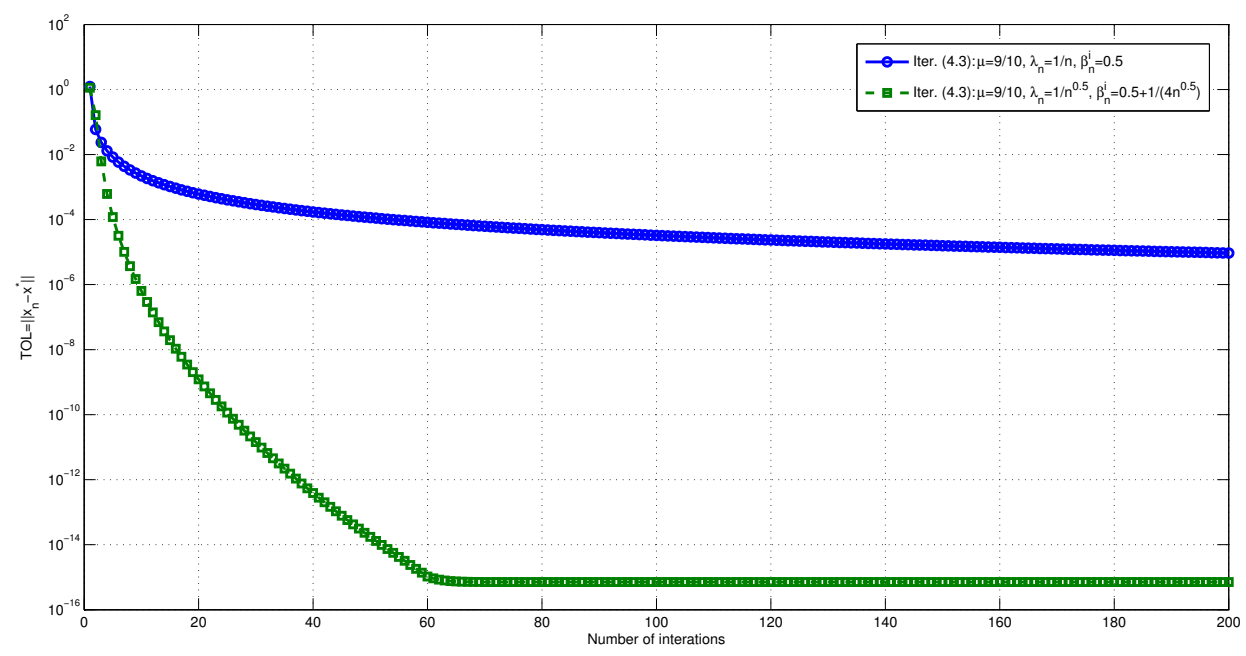

FIGURE 1.

b) Numerical results for Algorithm 3.2

- Applying iterative process (4.4) with $\mu=9 / 10, \beta_{n}^{i}=1 / 2$ and $\lambda_{n}=1 / n$ for all $n \geq 1$ and for all $i=1,2, \ldots, N$, and $x^{0}=(3,4,5)$, we obtain the following table of results:

\begin{tabular}{llll}
\hline TOL & $\left\|x^{n}-x^{*}\right\|$ & $n$ & $x^{n}$ \\
\hline \hline $10^{-5}$ & $9.97 \times 10^{-6}$ & 412 & $\left(-1.000004,9.99994 \times 10^{-1},-7.05 \times 10^{-6}\right)$ \\
$10^{-6}$ & $9.99 \times 10^{-7}$ & 1478 & $\left(-1.000000,9.99999 \times 10^{-1},-7.07 \times 10^{-7}\right)$ \\
$10^{-7}$ & $9.99 \times 10^{-8}$ & 5308 & $\left(-1.000000,9.99999 \times 10^{-1},-7.07 \times 10^{-8}\right)$ \\
$10^{-8}$ & $9.99 \times 10^{-9}$ & 19075 & $\left(-1.000000,9.99999 \times 10^{-1},-7.07 \times 10^{-9}\right)$ \\
$10^{-9}$ & $9.99 \times 10^{-10}$ & 68548 & $\left(-1.000000,9.99999 \times 10^{-1},-7.07 \times 10^{-10}\right)$ \\
\hline \multicolumn{5}{r}{ TABLE 3. Table of numerical results }
\end{tabular}

- Applying iterative process (4.4) with $\mu=9 / 10, \beta_{n}^{i}=1 / 2+1 / 4 \sqrt{n}$ and $\lambda_{n}=1 / \sqrt{n}$ for all $n \geq 1$ and 
for all $i=1,2, \ldots, N$ (note that in this case the sequences $\left\{\beta_{n}^{i}\right\}$ and $\left\{\lambda_{n}\right\}$ do not satisfy the conditions $\sum_{n=1}^{\infty}\left|\beta_{n+1}^{i}-\beta_{n}^{i}\right|<\infty$ for all $i=1,2, \ldots, N$ and $\left.\sum_{n=1}^{\infty}\left|\lambda_{n+1}-\lambda_{n}\right|<\infty\right)$, and $x^{0}=(3,4,5)$, we obtain the following table of results:

\begin{tabular}{llll}
\hline TOL & $\left\|x^{n}-x^{*}\right\|$ & $n$ & $x^{n}$ \\
\hline \hline $10^{-5}$ & $4.08 \times 10^{-6}$ & 9 & $\left(-1.000002,9.99997 \times 10^{-1},-2.90 \times 10^{-6}\right)$ \\
$10^{-6}$ & $8.05 \times 10^{-7}$ & 11 & $\left(-1.000000,9.99999 \times 10^{-1},-5.72 \times 10^{-7}\right)$ \\
$10^{-7}$ & $5.37 \times 10^{-8}$ & 15 & $\left(-1.000000,9.99999 \times 10^{-1},-3.82 \times 10^{-8}\right)$ \\
$10^{-8}$ & $9.59 \times 10^{-9}$ & 18 & $\left(-1.000000,9.99999 \times 10^{-1},-6.82 \times 10^{-9}\right)$ \\
$10^{-9}$ & $7.86 \times 10^{-10}$ & 23 & $\left(-1.000000,9.99999 \times 10^{-1},-5.59 \times 10^{-10}\right)$ \\
\hline \multicolumn{5}{c}{ TABLE 4. Table of numerical results }
\end{tabular}

The strong convergence of iterative process (4.4) is also described in Fig. 2.

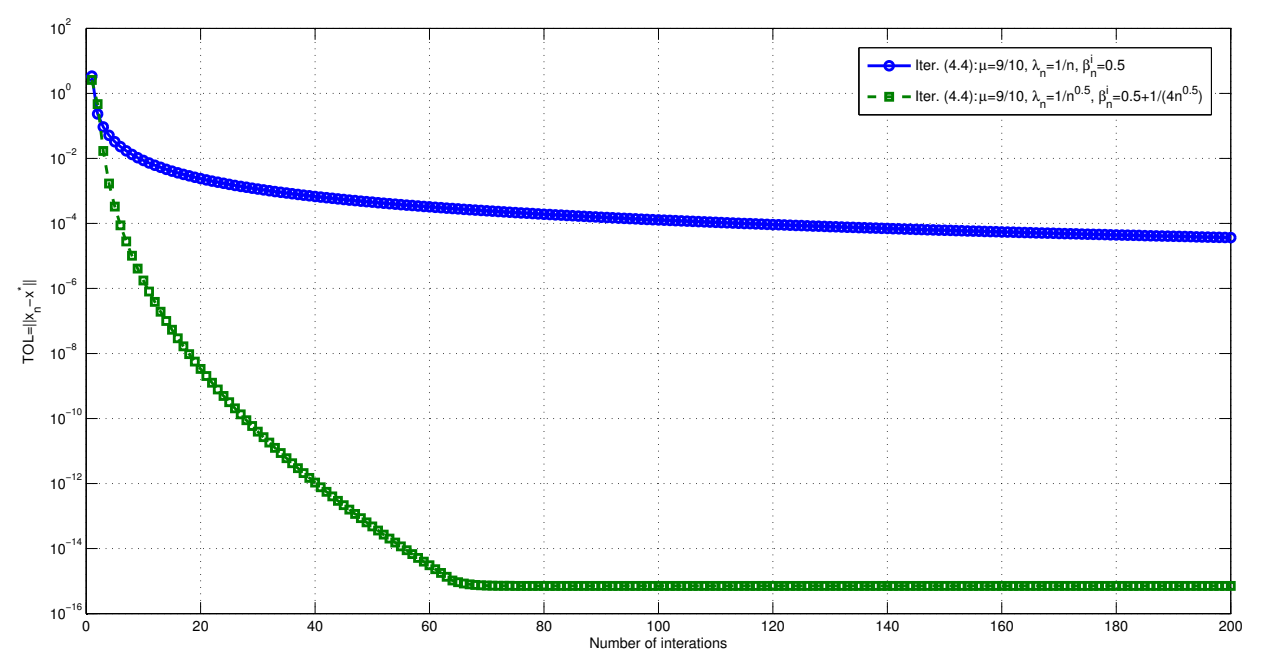

FIGURE 2.

\section{Acknowledgment}

The second author was supported by the Science and Technology Fund of Thai Nguyen University of Technology (TNUT).

\section{REFERENCES}

[1] K. Aoyama, H. Iiduka, W. Takahashi, Weak convergence of an iterative sequence for accretive operators in Banach spaces, Fixed Point Theory Appl. 2006 (2006), Article ID 35390.

[2] K. Aoyama, H. Iiduka, W. Takahashi, Strong convergence of Halpern's sequence for accretive operators in a Banach space, PanAmer. Math. J. 16 (2006), 97-104.

[3] V. Barbu, Th. Precupanu, Convexity and Optimization in Banach Spaces, Editura Academiei R. S. R., Bucharest, 1978.

[4] F.E. Browder, W.V. Petryshyn, Construction of fixed points of nonlinear mappings in Hilbert space, J. Math. Anal. Appl. 20 (1967), 197-228. 
[5] L.-C. Ceng, Q.H. Ansari, J.-C. Yao, Mann-type steepset-descent and modified hybrid steepset-descent methods for variational inequality in Banach spaces, Numer. Funct. Anal. Optim. 29 (2008), 987-1033.

[6] J.C. Cho, X. Qin, Viscosity approximation methods for a family of $m$-accretive mapping in reflexive Banach spaces, Positivity 12 (2008), 483-494.

[7] J.S. Jung, Strong convergence of an iterative method for finding common zeros of a finite family of accretive operators, Commun. Korean Math. Soc. 24 (2009), 381-393.

[8] J.K. Kim, T.M. Tuyen, Viscosity approximation method with Meir-Keeler contractions for common zero of accretive operators in Banach spaces, Fixed Point Theory Appl. 2015 (2015), Article ID 9.

[9] D. Kinderlehrer, G. Stampacchia, An Introduction to Variational Inequalities and Their Applications, Academic Press, New York, 1980.

[10] P.E. Maingé, Strong convergence of projected subgradient methods for nonsmooth and nonstrictly convex minimization, Set-Valued Anal. 16 (2008), 899-912.

[11] X. Qin, S.Y. Cho, L. Wang, Iterative algorithms with errors for zero points of m-accretive operators, Fixed Point Theory Appl. 2013 (2013), Article ID 148.

[12] S. Reich, Book Review: Geometry of Banach Spaces, Duality Mappings and Nonlinear Problems, Bull. Amer. Math. Soc. 26 (1992), 367-370.

[13] S. Reich, On the asymptotic behavior of nonlinear semigroups and the range of accretive operators, J. Math. Anal. Appl. 79 (1981), 113-126.

[14] T.M. Tuyen, Strong convergence theorem for a common zero of $m$-accretive mappings in Banach spaces by viscosity approximation methods, Nonlinear Funct. Anal. Appl. 17 (2012), 187-197.

[15] L. Wei, R.P. Agarwal, Relaxed iterative methods for an infinite family of d-accretiv mappings in a Banach space with their applications, J. Nonlinear Funct. Anal. 2018 (2018), Article ID 16.

[16] H.K. Xu, Inequalities in Banach spaces with applications, Nolinear Anal. 16 (1991), 1127-1138.

[17] Z.Q. Xue, H.Y. Zhou, Y.J. Cho, Iterative solutions of nonlinear equations for m-accretive operators in Banach spaces, J. Nonlineaer Convex Anal. 1 (2000), 313-320.

[18] Y. Yamada, The hybrid steepest-descent method for variational inequalities problems over the intersection of the fixed point sets of nonexpansive mappings, In: Butnariu, D., Censor, Y., Reich, S. (eds.) Inhently Parallel Algorithms in Feasibility and Optimization and Their Applications, North-Holland, Amsterdam (2001), 473-504.

[19] H. Zegeye, N. Shahzad, Strong convergence theorems for a common zero of a finite family of $m$-accretive mappings, Nonlinear Anal. 66 (2007), 1161-1169. 